\title{
A New Method to Fabricate Metal Tips for Scanning Probe Microscopy
}

\author{
member Takayuki Yagi, Yasuhiro Shimada, Tsutomu Ikeda, \\ Osamu Takamatsu, Kiyoshi Takimoto, and Yutaka Hirai*
}

Canon Research Center, Canon Inc., ${ }^{*}$ Canon Research Center America, Inc.

\begin{abstract}
Summary
We present a new method to fabricate sharp metal tips on cantilevers for a SPM. The metal tip, which is deposited and patterned as a film on a silicon mold with pyramidal etch pits, is attached by metal-to-metal bonding to a metal pad on a substrate. Then the tip on the substrate is peeled off the mold at room temperature. The tip surface is very smooth without grain boundaries associated with deposited thin films. A platinum tip with a radius curvature of less than $15 \mathrm{~nm}$ was successfully fabricated. In addition, the mold can be reused because the mold is not dissolved during the tip fabrication. By applying this method in which the tip fabrication process is independent of the cantilever process, we succeeded to form a tip on a piezoresistive cantilevers. Moreover, we used the cantilever with the tip in a piezoresistive AFM and an AFM/STM apparatus and obtained high resolution topography and surface conductance images, respectively.
\end{abstract}

Key words: tip, SPM, cantilever, peeling, mold

\section{Introduction}

In recent years, various types of scanning probe microscopes (SPM) have been researched. Using SPMs, one obtains a surface image by detecting various interactions between a fine tip and a sample surface. Among SPMs, an atomic force microscope combined with a scanning tunneling microscope (AFM/STM) apparatus using a conductive cantilever with a conductive tip is very useful for us because we can obtain topography and surface conductance images of the sample simultaneously and independently [1]. In order to achieve high resolution, it is crucial to fabricate a sharp conductive tip on a cantilever.

There are several methods for forming a tip. A method of forming currently commercially available cantilevers is the process of a pyramidal $\mathrm{Si}_{3} \mathrm{~N}_{4}$ tip formation by dissolving a silicon mold $[2,3]$. Previously reported processes involve wet, dry, or a combination of both dry and wet etching techniques, besides oxidation, to provide silicon tips [4-8], silicon dioxide tips [2], and polysilicon tips [9]. In order to use the tips in the AFM/STM, a conductive film is deposited on them. Because grain boundaries of the conductive film appear at the point of the tips, it is difficult to control the point shape and obtain a stable tunneling current. Using the direct electron-beam deposition process [10], a very high aspect ratio nanometer-scale conductive tip can be deposited on cantilevers [11], but this process is low in throughput and not a batch process. Small metal cones grown by an evaporation technique [12] can be formed on cantilevers [2]. However, the yield of this process is low because tip materials and process tolerances are severely restricted.

In this paper, we describe a new method to fabricate metal tips without grain boundaries for an AFM/STM. Using this method, metal tips can be attached to various cantilevers with batch fabrication at room temperature.

\section{Fabrication}

The fabrication process for these metal tips is described below. In this process, typical tip materials are platinum $(\mathrm{Pt})$ and gold $(\mathrm{Au})$. These materials are very suitable for use as a conductive tip of a STM because they do not form a native oxide layer. One typical example of the process sequence using $\mathrm{Pt}$ as tip material is shown in Fig. 1. Initially, a $100 \mathrm{~nm}$-thick thermally grown $\mathrm{SiO}_{2}$ film is formed on a double side polished Si (100) wafer and patterned to form openings with the size of $10 \mu \mathrm{m}$ square by photolithography and buffered hydrofluoric acid (BHF) etching. A pyramidal etch pit terminated by intersecting (111) planes is formed in the opening by anisotropic etching which uses a potassium hydroxide $(\mathrm{KOH})$ aqueous solution $\left(27 \%\right.$ by weight) at $90{ }^{\circ} \mathrm{C}$. Next, the $\mathrm{SiO}_{2}$ film is removed using a BHF. Thereafter a 500 nm-thick thermally grown $\mathrm{SiO}_{2}$ film is formed on the $\mathrm{Si}$ 
wafer with pyramidal etch pits as a mold. A $500 \mathrm{~nm}$-thick $\mathrm{Pt}$ film as a metal tip film is sputtered onto the $\mathrm{SiO}_{2}$ film and patterned into the shape of a tip by photolithography and reactive ion etching (RIE) (Fig. 1(a)). $\mathrm{A} \mathrm{Si}_{3} \mathrm{~N}_{4}$ film is deposited on another Si (100) substrate by low-pressure chemical vapor deposition (LPCVD). A $50 \mathrm{~nm}$-thick Ti layer and a $100 \mathrm{~nm}$-thick Au layer are subsequently Ebeam deposited on the $\mathrm{Si}_{3} \mathrm{~N}_{4}$ layer and patterned to form a metal pad (Fig. 1(b)). The Ti layer is necessary to increase the adhesion of Au. Using a bonding apparatus shown in Fig. 2, the mold and the Si substrate are aligned and the metal tip film is bonded to the metal pad by pressing the mold and the Si substrate together with a force in the range $0.5-5 \times 10^{9} \mathrm{~N} / \mathrm{m}^{2}$ at room temperature (Fig. 1(c)). The bonding apparatus consists of an infrared light source, a half mirror, $\mathrm{z} \theta$-stage, an infrared camera for alignment, holders for fixing a mold and a substrate with vacuum, and a pressure monitor for detecting a force. Separating the mold and the substrate, we peel the metal tip film off the $\mathrm{SiO}_{2}$ film and attach a Pt tip, having a cavity, to the metal pad firmly (Fig. 1(d)). We make use of metal (Pt)-to-metal ( $\mathrm{Au}$ ) bonding and poor adhesion due to low-surface energy of $\mathrm{SiO}_{2}$ against $\mathrm{Pt}$. The cavity makes the tip lighter in weight, while retaining sufficient stability. Figure 3 shows a magnified SEM view of the point of a Pt tip with a radius of tip curvature less than 15 nm measured from the micrograph. The surface of our tips is very smooth without grain boundaries associated with deposited thin films and its shape is very acute, because the back side of the metal tip film becomes the tip surface.

An annealing process is occasionally needed for device formations after the tip fabrication. Then, we investigated the deformation of $\mathrm{Pt}$ tips in shape after annealing at $200{ }^{\circ} \mathrm{C}$ and $350{ }^{\circ} \mathrm{C}$ for $1 \mathrm{~h}$. It was found that the tip radius did not change at these annealing temperatures.

Before concluding this section, we should note that one of the important features of the method is to form tips without dissolving the $\mathrm{Si}$ mold. We investigated the possibility of reusing the mold. An Au tip with a radius curvature of less than $20 \mathrm{~nm}$ was formed following the same procedure as that in Fig. 1 , except that a $1 \mu \mathrm{m}$ thick Au film was evaporated and patterned as metal tip film. Next, the mold used was cleaned by using an aqueous solution mixture of iodine $\left(\mathrm{I}_{2}\right)$ and potassium iodide (KI). A $1 \mu \mathrm{m}$-thick Au film was evaporated onto this mold again and an Au tip was formed by the same procedure. Again, the tip radius was less than $20 \mathrm{~nm}$ equal to that of the tip formed the first time. This demonstrates that the mold can be reused, resulting in high productivity and low manufacturing cost [13].

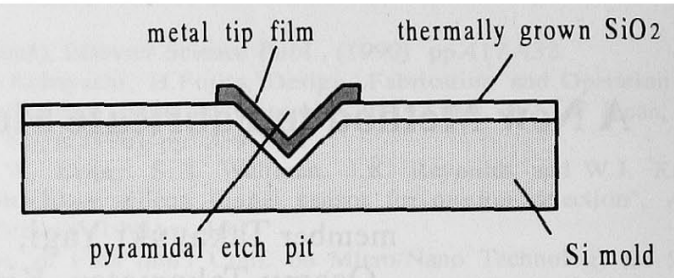

(a) Formation of Si mold and Tip film
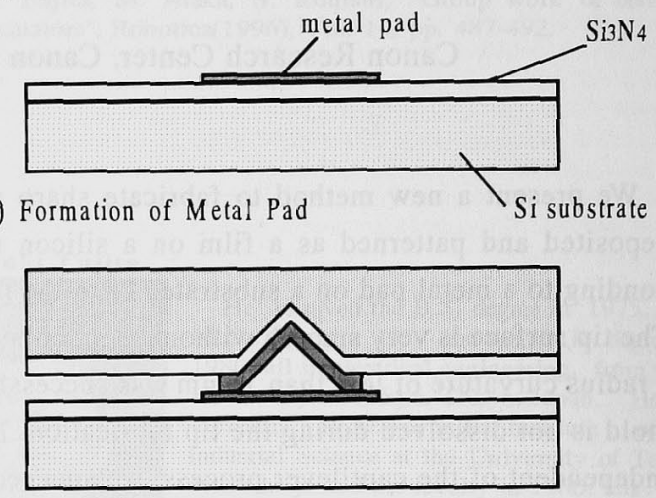

(c) Alignment and Bonding

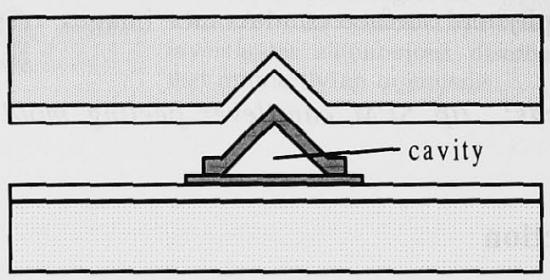

(d) Peel off

Figure 1. Process sequence for the fabrication of a metal tip.

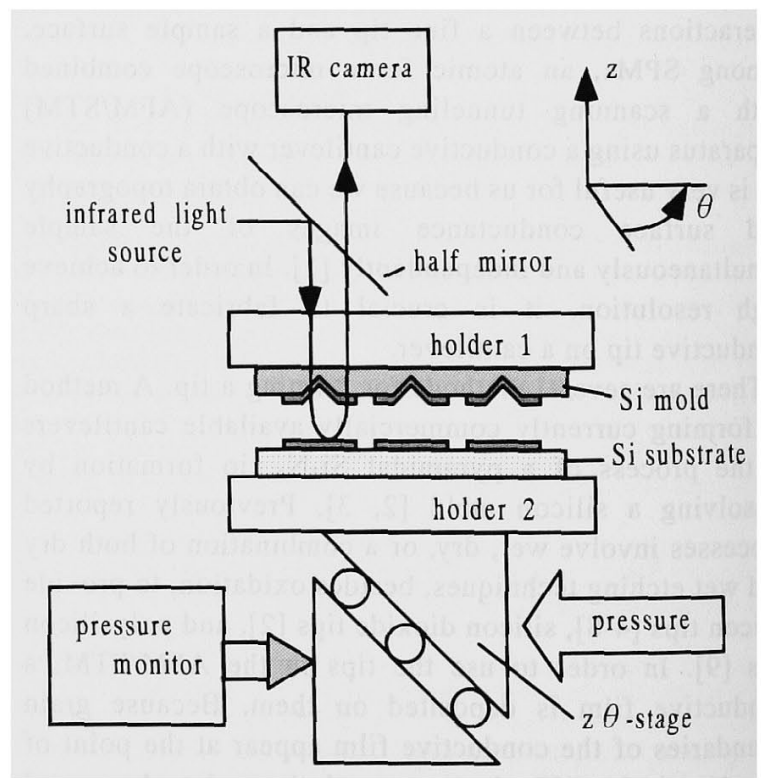

Figure 2. Schematic diagram of the bonding apparatus. 


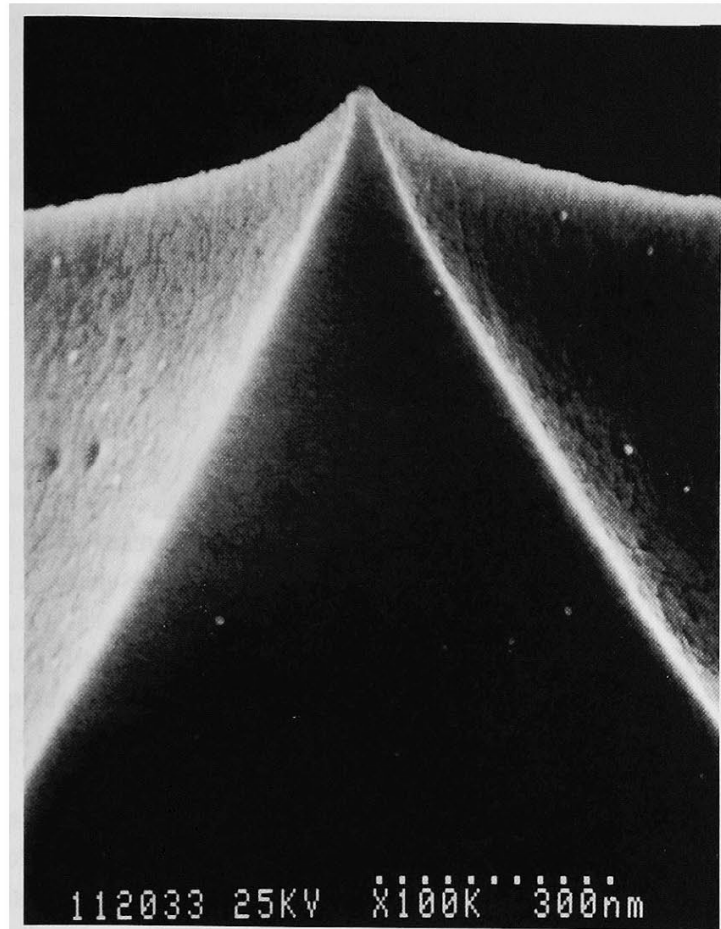

Figure 3. SEM micrograph of the point of a Pt tip with a tip radius of less than $15 \mathrm{~nm}$.

\section{Applications}

\subsection{Tip attachment on piezoresistive cantilevers}

Using this method, we can fabricate metal tips on various cantilevers in a batch process. We demonstrate one typical example of a Pt tip attached to a silicon piezoresistive cantilever. A piezoresistive cantilever is a promising tool for an AFM because it requires no optical sensing unit and can simplify AFM systems [14, 15, 16]. Figure 4 shows the process sequence for tip attachment on a piezoresistive cantilever.

The starting material is a bond and etch back silicon on insulator (BESOI) wafer named ELTRAN ${ }^{\circledR}$ [17]. The top layer is made of p-type silicon, $0.05 \Omega \mathrm{cm}, 1233 \pm 21$ $\mathrm{nm}$-thick, and the insulator is a $325 \mathrm{~nm}$-thick oxide layer. A $200 \mathrm{~nm}$-thick $\mathrm{Si}_{3} \mathrm{~N}_{4}$ film is deposited on the backside of the wafer by LPCVD and is patterned to form an opening. Arsenic is implanted at $100 \mathrm{keV}, 5 \times 10^{14}$ ions $/ \mathrm{cm}^{2}$ and doped at $1000{ }^{\circ} \mathrm{C}$. The top layer is patterned into the shape of a cantilever and a resistor is formed. Phosphosilicate glass (PSG) is deposited on the cantilever and patterned to form contact holes. An aluminum (Al) film is E-beam deposited, patterned to form an electrode and annealed to provide contacts to the resistor. A $50 \mathrm{~nm}$ thick $\mathrm{Ti}$ layer and a $100 \mathrm{~nm}$-thick $\mathrm{Au}$ layer are subsequently E-beam deposited and patterned into the shape of a metal pad (Fig. 4(a)). Using the same procedure as that in Fig. 1, we form a $\mathrm{Pt}$ film on a $\mathrm{Si}$ mold. Through aligning, bonding and peeling, we mount Pt tips on the cantilevers (Fig. 4(b),(c)). A resin film is spun onto the front of the wafer. The $\mathrm{Si}$ substrate is etched from the back using $\mathrm{KOH}$ solution at $95{ }^{\circ} \mathrm{C}$ and the oxide layer under the cantilevers is removed using a BHF from the back. The resin film is removed so that the cantilevers with the Pt tips are released (Fig. 4(d)). Figure 5 are SEM micrographs of piezoresistive cantilevers with Pt tips with radii of tip curvature less than $15 \mathrm{~nm}$. The measured resonant frequency of the cantilever is $14 \mathrm{kHz}(17 \mathrm{kHz}$ without a tip) and the calculated spring constant of it is $0.1 \mathrm{~N} / \mathrm{m}$. The measured sensitivity of the cantilever in terms of $\Delta R / R$ is $3 \times 10$ $7 / \mathrm{nm}$. Figure 6 shows a piezoresistive image of an $\mathrm{Au}$ film, which was E-beam deposited, obtained with this cantilever in contact mode. We clearly mapped grain boundaries of the Au film with this tip.

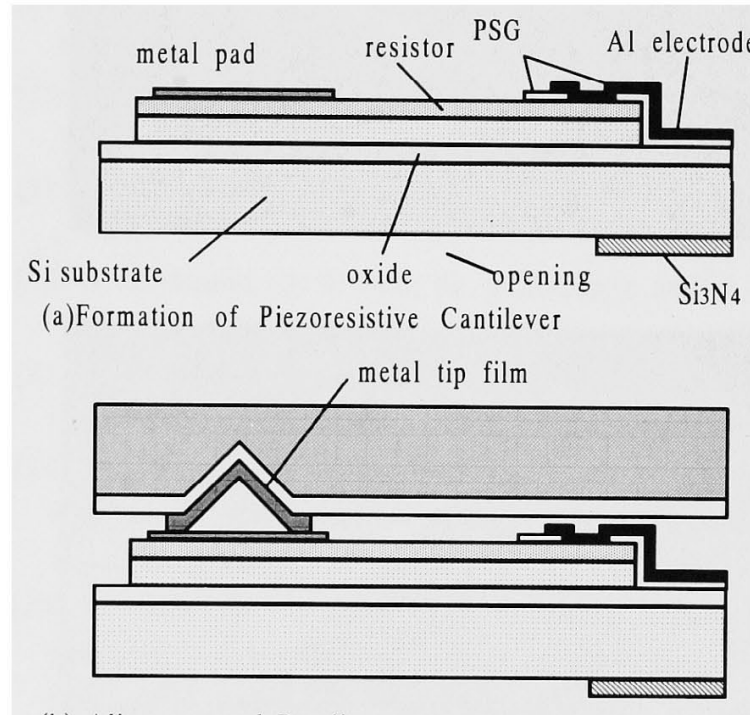

(b) Alignment and Bonding

(c) Peel off
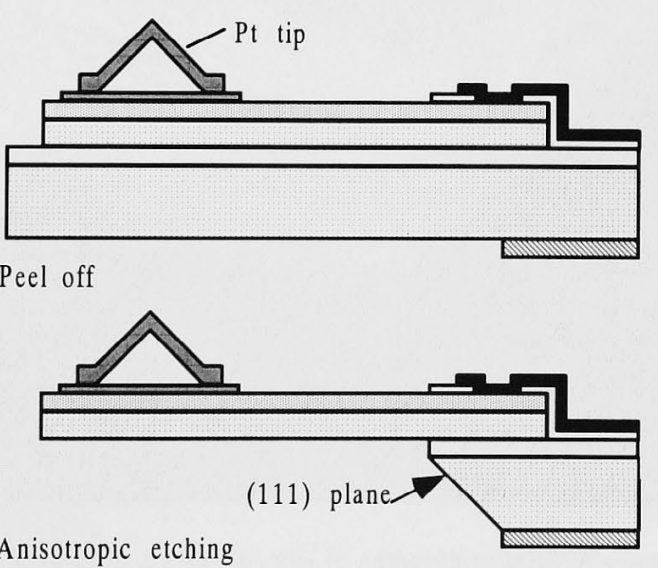

(d) Anisotropic etching

Figure 4. Process sequence for the fabrication of piezoresistive cantilvers with Pt tips. 

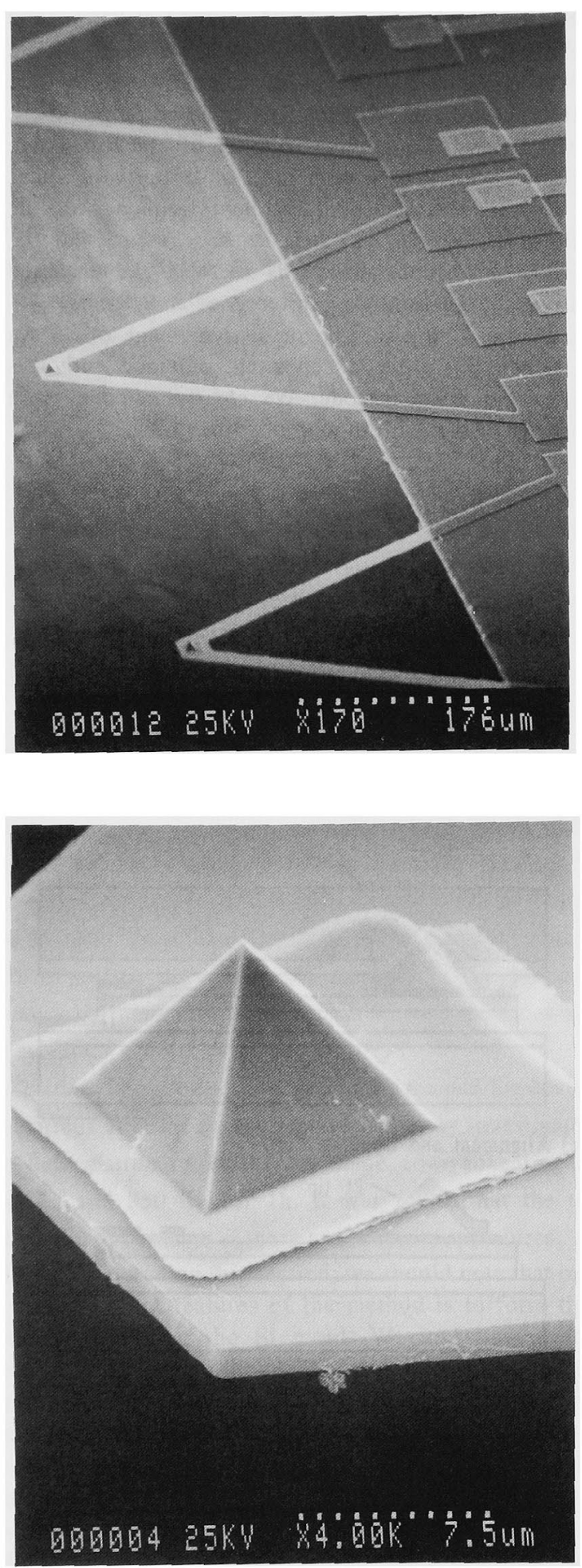

Figure 5. SEM micrograph of silicon piezoresistive cantilevers with Pt tips.

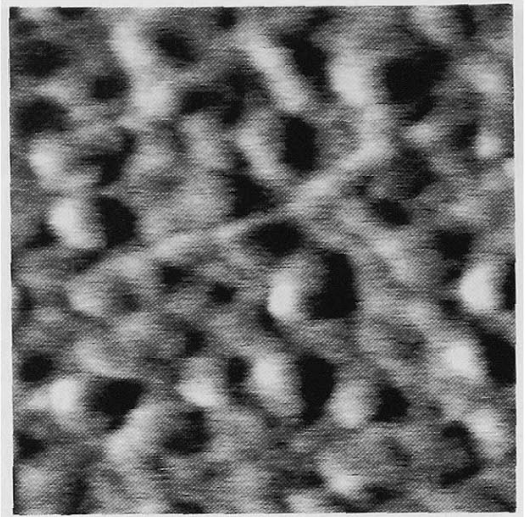

Figure 6. Image of an Au film taken with a piezoresistive cantilever with a Pt tip. The scan area is $500 \mathrm{~nm}$-square.

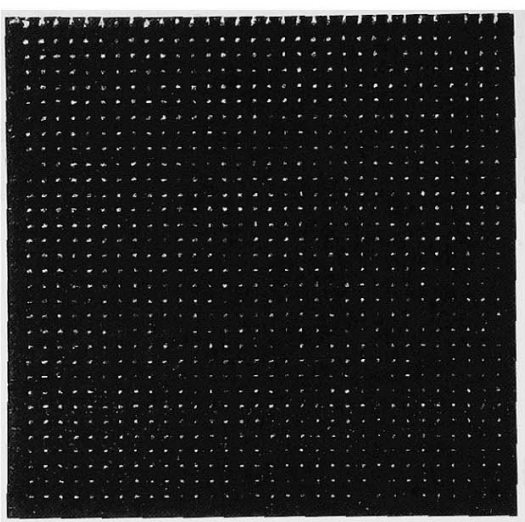

Figure 7. Current image of the LB film. The scan area is $2 \mu \mathrm{m}$-square.

\subsection{AFM/STM cantilever for information storage}

There are several applications using AFM/STM cantilevers, such as information storage [18, 19] and scanning probe lithography $[20,21]$. We have already reported writing and reading bit arrays for information storage using an AFM/STM apparatus and a medium consisting of a polyimide (PI) Langmuir-Blodgett (LB) film on an Au substrate [22]. These bit arrays can be formed by application of voltage pulses with an AFM/STM cantilever and exhibit a change in conductance only with no change in topography [23].

We used silicon piezoresistive cantilevers with Pt tips, as described before to write such bit arrays. The integrated resistors were used as conductors for the tip current only and an AFM image was obtained by the optical lever method. The diameter of a bit depends on the radius of the tip. We used a six-layer PI LB film coated onto an Au (111) surface as a medium. Figure 7 shows the current image after applying $+13 \mathrm{~V}, 0.2 \mu \mathrm{s}$ rectangular voltage pulses $(32 \times 32$ pulses $)$. The bias 
voltage applied to the sample to acquire the current image was $+3.3 \mathrm{~V}$. In the current image, clear small bits about $10 \mathrm{~nm}$ in diameter were observed. Also we found that the radius of tip curvature did not change before and after this experiment from the micrograph. As is evident, we can obtain high resolution current images and small bits with metal tips fabricated with the new method.

\section{Conclusion}

We have presented a new method to fabricate sharp, uniform and reproducible metal tips on cantilevers in a batch process. The method has the following advantages:

(1) Metal tips without grain boundaries.

(2) The tip fabrication process is independent of the cantilever process.

(3) The tip mounting process onto the cantilevers takes place at room temperature.

(4) The mold for tip fabrication can be reused.

We used a cantilever with one of these metal tips in a piezoresistive AFM and an AFM/STM apparatus and obtained high resolution topography images and current image. This method will be applied to tip fabrication on micro-actuators and a variety of demands for sharp metal tips.

\section{Acknowledgments}

We wish to acknowledge the help of our Device Development Center, Canon Inc. for providing the BESOI wafers, and express our gratitude to Mr. Shunichi Shido and Dr. Masafumi Kyogaku for supplying the topography and current images.

(Received Feb. 24, 1997 )

\section{References}

[1] Y. Sugawara, T. Ishizaka, S. Morita, S. Imai, and Nobuo Mikoshiba, J. J. Appl. Phys. 29, L 157 (1990)

[2] T. R. Albrecht, S. Akamine, T. E. Carver, and C. F. Quate, J. Vac. Sci. Technol. A 8, 3386 (1990)

[3] S. Akamine, and C. F. Quate, J. Vac. Sci. Technol. B 10, 2307 (1992)

[4] S. Akamine, R. C. Barrett, and C. F. Quate, Appl. Phys. Lett. 57, 316 (1990)

[5] O. Wolter, Th. Bayer, and J. Greschner, J. Vac. Sci. Technol. B 9, 1353 (1991)

[6] R. A. Buser, J. Brugger, C. Linder, and N. F. de Rooij, Transducers ‘91, pp.249-252

[7] Y. Xu, S. A. Miller, and N. C. MacDonald, Transducers ' 95 • Eurosensors IX, pp.640-643

[8] A. Boisen, O. Hansen, and S. Bouwstra, J. Micromech. Microeng. 6, 58 (1996)
[9] L. C. Kong, B. G. Orr, and K. D. WISE, J. Vac. Sci. Technol. B 11, 634 (1993)

[10] K. L. Lee, and M. Hatzakis, J. Vac. Sci. Technol. B7, 1941 (1989)

[11] K. L. Lee, D. W. Abraham, F. Secord, and L. Landstein, J. Vac. Sci. Technol. B 9, 3562 (1991)

[12] C. A. Spindt, I. Brodie, L. Humphrey and E. R. Westerberg, J. Appl. Phys. 47, 5248 (1976)

[13] T. Yagi, Y. Shimada, T. Ikeda, O. Takamatsu, H. Matsuda, K. Takimoto, and Y. Hirai, Proc. of MEMS'97, 129 (1997)

[14] M. Tortonese, H. Yamada, R. C. Barrett, and C. F. Quate, Transducers `91, pp.448-451

[15] M. Tortonese, R. C. Barrett, and C. F. Quate, Appl. Phys. Lett. 62, pp834-836 (1993)

[16] B. W. Chui, T. D. Stowe, and T. W. Kenny, Appl. Phys. Lett. 69, 2767 (1996)

[17] T. Yonehara, K. Sakaguchi, and N. Sato, Appl. Phys. Lett. 64, 2108 (1994)

[18]R. C. Barrett, and C. F. Quate, J. Appl. Phys. 70, 2725 (1991)

[19] H. Kado, and T. Tohda, Appl. Phys. Lett. 66, 2961 (1995)

[20] S. C. Minne, H. T. Soh, Ph. Flueckiger, and C. F. Quate, Appl. Phys. Lett. 66, 703 (1995)

[21] S. C. Minne, S. R. Manalis, A. Atalar, and C. F. Quate, J. Vac. Sci. Technol. B 14, 2456 (1996)

[22] K. Yano, R. Kuroda, Y. Shimada, S. Shido, M. Kyogaku, H. Matsuda, K.Takimoto, K. Eguchi, and T. Nakagiri, J. Vac. Sci. Technol. B 14, 1353 (1996)

[23] K. Yano, M. Kyogaku, Y. Shimada, S. Shido, H. Matsuda, K.Takimoto, O. Albrecht, K. Eguchi, and T. Nakagiri, Appl. Phys. Lett. 68, 188 (1996)

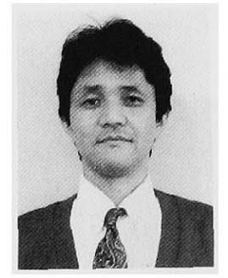

Takayuki Yagi (member) He received the B.S. degree in 1981 from Sophia University, Tokyo, Japan and the M.S. degree in 1983 from Tokyo Institute of Technology, Tokyo, Japan. He joined Canon Inc. in 1983.

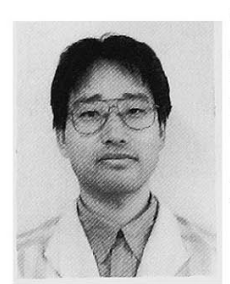

Yasuhiro Shimada (non-member) He received the B.S. degree in 1987 and the M.S. degree in 1989 from Waseda University, Tokyo, Japan. He joined Canon Inc. in 1989. 
Tsutomu Ikeda (non-member)

$\mathrm{He}$ received the B.S. degree in 1983 and the M.S. degree in 1985 from Saitama University, Saitama, Japan. He joined Canon Inc. in 1985.

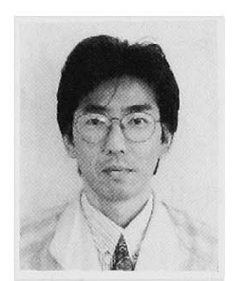

Osamu Takamatsu (non-member)

He graduated Kanagawa National school of commerce and technology., Kanagawa, Japan in 1979. He joined Canon Inc. in 1979.

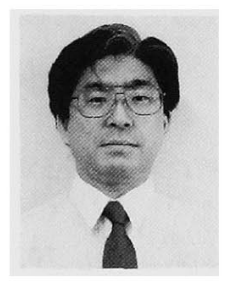

Kiyoshi Takimoto (non-member)

He received the B.S. degree in 1980 , the M.S. degree in 1982 from Kyoto University, Kyoto, Japan. He joined Canon Inc. in 1985.

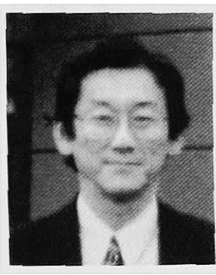

Yutaka Hirai (non-member)

He received the B.S. degree in 1971, the M.S. degree in 1973 and the Ph.D degree in 1976 from Sophia University, Tokyo, Japan. He joined Canon Inc. in 1977. He has been the president of Canon Research Center America, Inc., California, USA since 1997. 